\title{
Complete Nucleotide Sequence of Artichoke latent virus Shows it to be a Member of the Genus Macluravirus in the Family Potyviridae
}

\author{
S. A. Minutillo, A. Marais, T. Mascia, C. Faure, L. Svanella-Dumas, S. Theil, A. Payet, \\ S. Perennec, L. Schoen, D. Gallitelli, and T. Candresse
}

First, third, and tenth authors: Dipartimento di Scienze del Suolo della Pianta e degli Alimenti, Università degli Studi di Bari Aldo Moro, Via Amendola 165/A, 70126 Bari, Italy; second, fourth, fifth, sixth, seventh, and eleventh authors: INRA, UMR 1332 BFP, CS20032, 33882 Villenave d'Ornon Cedex, France and Université de Bordeaux, UMR 1332 BFP, CS20032, 33882 Villenave d'Ornon Cedex, France; eighth author: Chambre d'Agriculture du Finistère, 29250 Saint Pol de Léon, France; and ninth author: Sudexpé-Sica Centrex, 66440 Torreilles, France.

Accepted for publication 3 March 2015.

\begin{abstract}
Minutillo, S. A., Marais, A., Mascia, T., Faure, C., Svanella-Dumas, L., Theil, S., Payet, A., Perennec, S., Schoen, L., Gallitelli, D., and Candresse, T. 2015. Complete nucleotide sequence of Artichoke latent virus shows it to be a member of the genus Macluravirus in the family Potyviridae. Phytopathology 105:1155-1160.

Complete genomic sequences of Artichoke latent virus (ArLV) have been obtained by classical or high-throughput sequencing for an ArLV isolate from Italy (ITBr05) and for two isolates from France (FR37 and FR50). The genome is 8,278 to 8,291 nucleotides long and has a genomic organization comparable with that of Chinese yam necrotic mosaic virus (CYNMV), the only macluravirus fully sequenced to date. The cleavage sites of the viral

polyprotein have been tentatively identified by comparison with CYNMV, confirming that macluraviruses are characterized by the absence of a P1 protein, a shorter and N-terminally truncated coat protein $(\mathrm{CP})$. Sequence comparisons firmly place ArLV within the genus Macluravirus, and confirm previous results suggesting that Ranunculus latent virus (RALV), a previously described Macluravirus sp., is very closely related to ArLV. Serological relationships and comparisons of the CP gene and of the partial RaLV sequence available all indicate that RaLV should not be considered as a distinct species but as a strain of ArLV. The results obtained also suggest that the spectrum of currently used ArLV-specific molecular hybridization or polymerase chain reaction detection assays should be improved to cover all isolates and strains in the ArLV species.
\end{abstract}

Globe artichoke (Cynara cardunculus var. scolymus) is a perennial plant grown for its large, fleshy, immature inflorescences (flower heads or capitula), which are appreciated for their taste and nutritional properties. Production is mainly concentrated in Mediterranean countries, among which Italy, France, Spain, and Egypt rank first, but extensive cropping also takes place in the Near East, South America (mainly Peru and Argentina), the United States, and China.

Traditional artichoke types are multiplied vegetatively by farmers, through offshoots and ovuli, without any active selection for sanitary status. This has led to the accumulation over time of a range of systemic pathogens, such as vascular fungi (mainly Verticillium spp.) and viruses. To date, 25 viruses belonging to 15 genera in five families have been isolated from globe artichoke (Gallitelli et al. 2012) and, among them, Artichoke latent virus (ArLV) is one of the most common. As suggested by its name, ArLV infections are generally symptomless. Reports of symptomatic ArLV infections also exist but generally involve mixed infection and synergistic effects with other viruses such as Artichoke Italian latent virus (AILV) and Tomato spotted wilt virus (Acquadro et al. 2010; Gallitelli et al. 2012; Minutillo et al. 2012).

ArLV is transmitted by several aphid species, including Myzus persicae, Brachycaudus cardui, and Aphis fabae, in a nonpersistent manner and was initially assigned to the genus Potyvirus in the

Corresponding author: T. Mascia; E-mail address: tiziana.mascia@uniba.it

First and second authors contributed equally to this work and should be considered as co-first authors.

The sequences reported in the present manuscript have been deposited in the GenBank database under the accession numbers KF155694, KP405232, and KP405233.

http://dx.doi.org/10.1094/PHYTO-01-15-0010-R

(C) 2015 The American Phytopathological Society family Potyviridae (Adams et al. 2012; Rana et al. 1982). However, the taxonomic position of ArLV has recently been questioned (Ciuffo et al. 2011), on the basis of the sequence of a 5,929-nucleotide (nt) fragment of a virus isolated from artichoke plants in Liguria (northern Italy), denoted Cynara 42 (Cy42) (Ciuffo et al. 2011). Isolates related to $\mathrm{Cy} 42$ were also found at a very high rate in artichoke fields of Sardinia, Latium, and Liguria and representative isolates obtained included Cy46 from Sardinia and Cy49 and IFA235 from Latium (Ciuffo et al. 2011). The Cy42 sequence fragment showed $98 \%$ identity at the nucleotide level with a Ranunculus isolate of the macluravirus Ranunculus latent virus (RaLV) (Turina et al. 2006) and $80 \%$ nucleotide identity $(90 \%$ amino acid identity for the putatively encoded protein) with the only available sequence for ArLV (X87255, approximately $500 \mathrm{nt}$ ), corresponding to a region comprising part of the NIa and NIb coding sequences (Ciuffo et al. 2011). However, primers ArLV30F and ArLV500R derived from the ArLV sequence (Grieco and Gallitelli 1999) failed to amplify any RaLV-positive artichoke sample, although primer ArLV30F could amplify RaLV isolates when used in combination with an RaLVspecific primer (Ciuffo et al. 2011). A probe derived from the ArLV 500-nt fragment did not hybridize to any of the 101 artichoke samples tested (Ciuffo et al. 2011). A phylogenetic analysis performed using the 500-nt fragment suggested that ArLV, like RaLV, was likely a Macluravirus sp. and that the initial assignment of ArLV to the genus Potyvirus was probably incorrect. In the absence of more detailed sequence information on ArLV and considering some reported host range and capsid protein $(\mathrm{CP})$ size differences between the two agents, Ciuffo et al. (2011) were unable to unambiguously decide on the genus assignment of ArLV and asked whether RaLV should be considered as a strain of ArLV or a distinct agent. Therefore, they recommended complete molecular characterization of ArLV isolates in order to settle these issues. In the present article, we report 
on the complete nucleotide sequence of three ArLV isolates, one from a Apulian globe artichoke (southern Italy, isolate ITBr05) and two from France (FR37 from Britany and FR50 from the region close to the Spanish border on the Mediterranean). The analysis of these sequences unambiguously demonstrates that ArLV belongs to the genus Macluravirus and the comparison of ArLV and RaLV sequences clearly indicates that they belong to the same species.

\section{MATERIALS AND METHODS}

Viral isolates. Twenty samples of globe artichoke 'Brindisino' were collected in Apulia (southern Italy) and used for mechanical inoculation onto Nicotiana benthamiana, which reacted with mosaic and leaf blade distortion within 2 weeks postinoculation. ArLVinfected samples were identified by dot-blot hybridization, using a digoxigenin-labeled DNA probe synthesized and used as described previously (Minutillo et al. 2012).

Symptomatic globe artichoke samples showing leaf curling and necrotic reactions were also obtained from Britany (northwestern France, sample FR37 from the Camus de Bretagne variety) and from the Mediterranean region close to the Spanish border (sample FR50, Sambo variety). Molecular hybridization with the ArLV probe (Minutillo et al. 2012) gave a clear positive signal for sample FR50 but no hybridization signal could be obtained with sample FR37, even when reducing the stringency of the hybridization conditions (not shown).

An RaLV isolate was obtained from Dr. Massimo Turina (IPSPCNR, Turin, Italy) and grown on plants of $N$. benthamiana, where it induced systemic mosaic and leaf distortion within 2 weeks after inoculation, as reported by Ciuffo et al. (2011).

Production of an AILV-free ArLV isolate. To eliminate AILV contamination from Italian samples with mixed infections, sap from $N$. benthamiana extracted in phosphate-buffered saline (PBS) buffer (10× PBS: $1.37 \mathrm{M} \mathrm{NaCl}, 27 \mathrm{mM} \mathrm{KCl}, 81 \mathrm{mM} \mathrm{Na}_{2} \mathrm{HPO}_{4}$, and $19 \mathrm{mM} \mathrm{KH}_{2} \mathrm{PO}_{4}, \mathrm{pH} 7.4$ ) was centrifuged briefly to spin-down leaf debris and $100 \mu \mathrm{l}$ of the supernatant mixed with $100 \mu \mathrm{l}$ of an antiserum was raised against AILV (Savino et al. 1977) and incubated at $37^{\circ} \mathrm{C}$ for $30 \mathrm{~min}$. After incubation, the mixture was centrifuged and two-thirds of the supernatant was transferred quickly to a fresh tube, without disturbing the pellet. The incubation was repeated twice and final supernatant was used for mechanical inoculation onto $N$. benthamiana, thus providing the ITBr05 isolate used in the present study. Absence of AILV in infected plants was verified by dot-blot hybridization and polymerase chain reaction (PCR), as described (Minutillo et al. 2012).

Immunosorbent electron microscopy. Immunosorbent electron microscopy (ISEM) was performed as described by Roberts and Harrison (1979) using an ArLV antiserum prepared by Rana et al. (1982) and an RaLV antiserum kindly provided by Dr. Massimo Turina (IPSP-CNR). Carbon-coated grids sensitized with ArLV or $\mathrm{RaLV}$ antibodies were floated overnight at $4^{\circ} \mathrm{C}$ on expressed sap of $N$. benthamiana leaves infected with ArLV-ITBr05 or with RaLV. After washing, the grids were floated for $15 \mathrm{~min}$ on drops of ArLV or

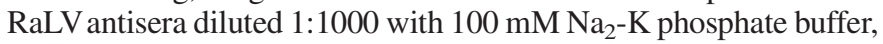
$\mathrm{pH} 7$, negatively stained with a $2 \%$ (wt/vol) aqueous solution of uranyl acetate, and observed with a Philips Morgagni 282D electron microscope.

Purification of ArLV particles and genome sequence determination. ArLV-ITBr05 was maintained in N. benthamiana and purified from systemically infected leaves harvested 2 weeks after inoculation, according to the method of Thompson et al. (1988). Viral RNA was extracted from purified virus particles, reverse-transcribed with random hexamers and oligo(dT) primer, and made double stranded using the cDNA Synthesis System (Roche, Germany) according to the manufacturer's instructions. The double-stranded cDNA was ligated into SmaI-digested, dephosphorylated pUC18 plasmid with T4 DNA ligase (Promega Corp., Madison, WI) and used to transform competent Escherichia coli DH5 $\alpha$ cells. Plasmids containing inserts of different size were identified and used for DNA automated sequencing in both directions (Primm, Milan, Italy). Additional pairs of primers were designed to obtain multiple overlapping cDNA clones to cover all the ArLV genome. The 3'-terminal sequence of the genomic RNA was determined from at least five different clones, while the $5^{\prime}$-end region was determined following instructions of the $5^{\prime} / 3^{\prime}$ rapid amplification of cDNA ends (RACE) kit, second generation (Roche, Germany), using $10 \mu \mathrm{M}$ primer RACE I (5'-GACTTCGAAATGCTTATCAACC-3') to synthesize the first cDNA strand and a second, nested primer RACE II (5'-GATTTTGAGTGCTGAACCCG-3') at $10 \mu \mathrm{M}$ for the PCR amplification. The final PCR product was ligated into a pGem-T Easy plasmid (Promega Corp.) and used to transform competent E. coli DH5 $\alpha$ cells and sequenced as described above.

Determination of ArLV genome sequence by high-throughput sequencing. Double-stranded (ds)RNA was extracted from leaves of the infected plants, reverse-transcribed, random amplified, and submitted to Illumina Hiseq (isolate FR37) or MiSeq (isolate FR50) multiplexed sequencing according to the strategy described by Candresse et al. (2013). Analysis of the sequence data were performed as previously reported (Candresse et al. 2013) using the CLC Genomics Workbench 6.5 (http://www.clcbio.com) and inhouse developed pipelines. Briefly, after demultiplexing, the qualitytrimmed reads were assembled de novo into contigs which were then annotated using BlastN and BlastX comparisons with the GenBank database using a $10^{-3} e$-value cut-off. Further assembly of the contigs into scaffolds was obtained by mapping them and residual reads, at reduced stringency, on the partial RaLV genome (HQ449546) or by aligning them on the genome of Chinese yam necrotic mosaic virus (CYNMV) (NC018455). When needed, primers were designed to amplify PCR products spanning the gaps between the various contigs or regions with low coverage and for which sequence confirmation was sought. The $5^{\prime}$ end of the genome of the FR37 isolate was determined by 5' RACE using the RACE-ArLV1: 5' CCTCCAC CATCAACCATGATAAATTCGCTCCC $3^{\prime}$ primer and following the recommendations of the RACE kit supplier (Takara Bio Europe/Clontech, Saint-Germain-en-Laye, France). All amplified fragments were submitted to Sanger sequencing (GATC Biotech, Mulhouse, France) either directly or after cloning in the pGEM-T Easy vector (Promega, Charbonnières-Les Bains, France).

Sequence comparisons and phylogenetic analyses. $\mathrm{Nu}-$ cleotide and putative protein sequences were analyzed using the software DNA Strider (Marck 1988), version 1.4. They were aligned with the ClustalW program (Thompson et al. 1994) (http://www.ebi. ac.uk/Tools/msa/clustalw2/) or implemented in Mega5.2 (Tamura et al. 2011). Average pairwise distances were computed using Mega 5.2. Phylogenetic reconstructions were performed using the neighborjoining technique with strict nucleotide or amino acid distances and randomized bootstrapping evaluation of branching validity using Mega 5.2.

\section{RESULTS}

Selection of ArLV isolates and genome sequence determination. Of 20 globe artichoke samples from the Apulia southern Italy region, 12 were found positive to ArLV using an ArLVspecific dot blot hybridization (Minutillo et al. 2012). As expected from results of a previous survey (Acquadro et al. 2010), all 12 ArLVpositive samples were also infected by AILV, as assessed by dot blot hybridization (Minutillo et al. 2012) and by observations with the electron microscope. The remaining eight samples, free from ArLV, were infected only by AILV, confirming previous observations that, for unknown reasons, the latter is by far the most predominant virus in Apulian globe artichoke fields (Acquadro et al. 2010; Gallitelli et al. 2012). Upon removal of AILV contamination (see Materials and Methods), ArLV was transmitted and grown in $N$. benthamiana, yielding ArLV isolate ITBr05, which was used for further characterization. Particles from crude sap extracts of $N$. benthamiana infected with the ITBr05 isolate could be trapped and decorated using an ArLVspecific antiserum in ISEM experiments, further confirming the 
identity of the ITBr05 isolate as ArLV (Fig. 1). However, the close links between ArLV and RaLV suggested by the work of Ciuffo et al. (2011) were confirmed by the trapping and decoration of these particles using an RaLV-specific antiserum and by the trapping and decoration of RaLV particles by the ArLV-specific antiserum (Fig. 1).

The ArLV-specific molecular hybridization assay was also performed on two symptomatic French artichoke samples, FR37 and FR50. A clear positive signal was obtained for sample FR50 but no hybridization signal could be obtained with sample FR37, even when reducing the stringency of the hybridization conditions (not shown). However, the FR37 viral isolate, in the absence of the coinfecting new Comoviridae sp. (see below), could be readily propagated in $N$. benthamiana and $N$. clevelandii, the latter being reported as a host for ArLV but not for RaLV (Ciuffo et al. 2011).

Determination of ArLV genome sequence. The genome sequence of the ITBr05 ArLV isolate was determined from overlapping cDNA clones obtained using genomic RNA extracted from viral particles purified from infected $N$. benthamiana. Gaps were closed using specific primers to amplify missing regions of the genomic RNA by PCR, and the extreme 5' part was determined by RACE experiments.

For isolates FR37 and FR50, the genomic sequence was obtained using high-throughput Illumina sequencing on random cDNAs prepared from dsRNA purified from the leaves of the original artichoke samples (Candresse et al. 2013). For isolate FR37, out of 103,399 Illumina reads of an average $73 \mathrm{nt}, 49,187$ corresponded to ArLV (47.6\%), providing an average coverage of $436 \times$. In the case of isolate FR50, coverage was lower because, out of 48,776 reads, $34,183(70.1 \%)$ corresponded to ArLV, giving an average coverage of $306 \times$. As for isolate ITBr05, gaps or low-coverage regions of the genome of isolates FR37 and FR50 for which sequence confirmation was sought were sequenced following PCR amplification of viral cDNAs with specific primers and a 5'RACE. During the assembly and contig annotation, evidence was obtained for the presence in the analyzed samples of a novel Comoviridae sp. (FR37 isolate) and a novel Alphaflexiviridae sp. (FR50 isolate) but there was no evidence for the presence of other agents belonging to the family Potyviridae besides the contigs and reads that were finally assembled in the ArLV genomic sequences (not shown). The novel Comoviridae and Alphaflexiviridae spp. will be described in detail elsewhere.

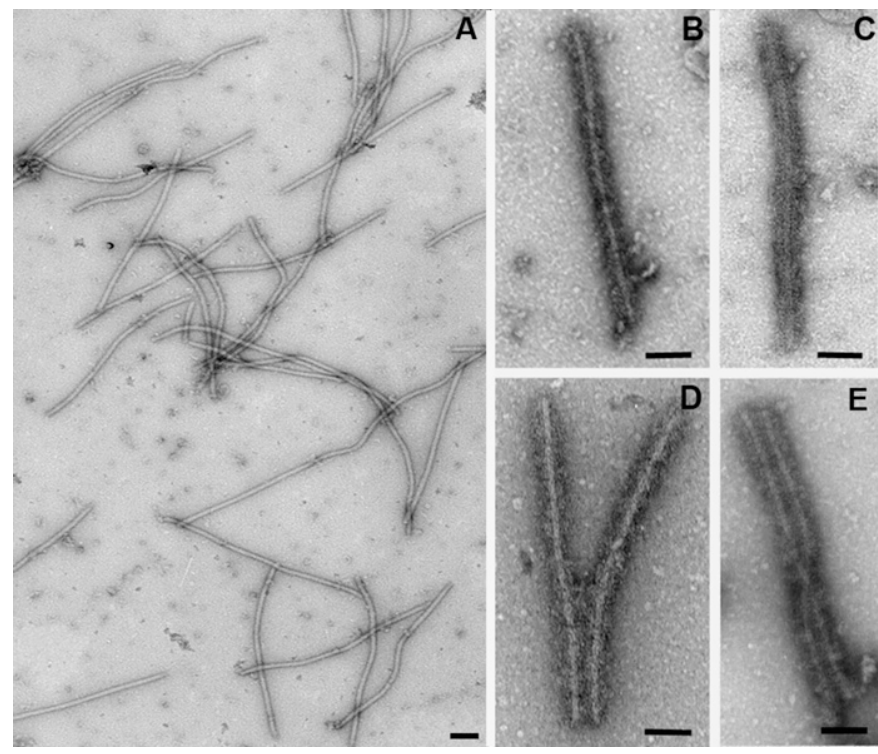

Fig. 1. Electron microscopy and immunosorbent electron microscopy of ArLV and RaLV particles. A, Negatively stained particles from a purified preparation of ArLV. Particles of $\mathbf{B}$ and $\mathbf{C}$, ArLV and $\mathbf{D}$ and $\mathbf{E}$, RaLV in crude extracts from Nicotiana benthamiana trapped and decorated with antisera to $\mathbf{B}$ and $\mathbf{D}, \mathrm{ArLV}$ and $\mathbf{C}$ and E, RaLV. Bars $=100 \mathrm{~nm}$.
Analysis of ArLV genome sequence. The completed ArLVITBr05 genome is 8,278 nt long, excluding the polyA tail (GenBank KF155694). By comparison, the sequence of the FR37 isolate is 8,287 nt long (GenBank KP405232) and that of the FR50 isolate (GenBank KP405233) is 8,291 nt long. Indel polymorphisms explaining these differences in size are observed both in the untranslated regions (UTR) and in the coding region. The $5^{\prime}$ and $3^{\prime}$ UTRs of ArLV-ITBr05 consist of $117 \mathrm{nt}$ (116 for FR37) and $245 \mathrm{nt}$ (246 for FR50 and 243 for FR37), respectively. As expected, in silico translation of the genomic sequence revealed a large open reading frame (ORF) of 7,914 nt for isolate ITBr05, which starts at the AUG at position 118 in the plant consensus sequence ACAAUGGC (Lütcke et al. 1987) and ends at termination codon UAG at position 8,034, covering $95 \%$ of the complete genome sequence. By comparison, the ORF encoded by isolates FR37 and FR50 is 7,926 nt long. The ORF of the three isolates translates into a polyprotein, whose putative cleavage sites yield nine functional products, denoted HC-Pro, P3, 7K, CI, 9K, $\mathrm{VPg}, \mathrm{NIa}, \mathrm{Nib}$, and $\mathrm{CP}$, on the basis of alignments with CYNMV (see below and Table 1), the only macluravirus for which a complete nucleotide sequence is available (AB710145). Similarly to CYNMV, the ArLV-ITBr05 genome lacks a putative P1 proteinase which, in other Potyviridae spp., is involved in the proteolytic cleavage of the polyprotein, together with the HC-Pro and NIa proteinases (Hull 2002). The HC-Pro domain has an estimated size of 261 amino acids (aa) and shares $48 \%$ identity with the HC-Pro of CYNMV (Table 1). HC-Pro cleaves itself from the P3 protein at the FVG/G cleavage site found at amino acid positions 259 to 262. As with CYNMV, five putative NIa proteinase cleavage sites were identified in ArLVITBr05, characterized by the dipeptide $Q(E) / M(A)$ with a conserved $\mathrm{L}$ residue at the -2 position from the cleavage site. The NIa-Pro cleavage sites were found between proteins P3-7K, 7K-CI, CI-9K, 9K-Nia, and NIb-CP at positions 562 (Q/A), $626(\mathrm{Q} / \mathrm{M}), 1,284(\mathrm{Q} / \mathrm{M}), 1,364(\mathrm{E} / \mathrm{M})$, and 2,325 $(\mathrm{Q} / \mathrm{M})$, respectively, of the polyprotein. These sites were replaced by noncanonical dipeptides $\mathrm{E} / \mathrm{V}(1,543)$ and $\mathrm{Q} / \mathrm{I}(1,760)$, which putatively cleave the VPg from the NIa domain and the NIa from the NIb, respectively. However, both sites had a conserved L residue at the -2 position. All cleavage sites were fully conserved in the sequences of the two French isolates. This gave proteins of 302 (P3), 65 (7K), 659 (CI), 81 (9K), 397 (VPg-NIa, 180 for VPg alone), $566(\mathrm{NIb})$, and $314(\mathrm{CP})$ amino acids, respectively, for the ITBr05 isolate, with only minor differences for the two French isolates. As for other Potyviridae members, a P3N-PIPO ORF (Chung et al. 2008) was detected, nested in the P3 ORF and starting with the consensus GAAAAAA sequence at positions 1,314 to 1,320 of the ITBr05 genome. This ORF was conserved in the French isolates and encoded a very short 56-aa protein that, like other P3N-PIPO, is expected to be expressed as a frameshift product fused to the $\mathrm{N}$-terminal part of $\mathrm{P} 3$. The most divergent proteins, when comparing with the FR37 and FR50 sequences, were the $\mathrm{CP}$ and the P3 (Table 1).

TABLE 1. Percent amino acid sequence identities between the various proteins of Artichoke latent virus (ArLV) isolate ITBr05 with those of ArLV isolates FR37 and FR50, those of Ranunculus latent virus (RaLV) isolate Cy42, and those of Chinese yam necrotic mosaic virus (CYNMV)

\begin{tabular}{lcccc}
\hline & \multicolumn{4}{c}{ Sequence identity $(\%)^{\mathrm{a}}$} \\
\cline { 2 - 5 } Gene & ArLV-FR37 & ArLV-FR50 & RaLV-Cy42 & CYNMV \\
\hline HC-Pro & 96.6 & 99.2 & n.a. & 48.3 \\
P3 & 83.8 & 86.7 & n.a. & 30.6 \\
PIPO & 87.5 & 98.2 & n.a. & 30.4 \\
7K & 100 & 100 & n.a. & 70.3 \\
CI & 95.1 & 96.7 & 91.7 & 53.1 \\
9K & 98.7 & 98.7 & 98.7 & 48.7 \\
VPg & 92.2 & 95.5 & 93.9 & 43.9 \\
NIa & 94.9 & 100 & 93.1 & 51.1 \\
NIb & 94.9 & 99.6 & 95.2 & 59.8 \\
CP & 80.8 & 98.7 & 81.4 & 45.1 \\
\hline
\end{tabular}

a Abbreviation: n.a. = not available (relevant sequence not available for isolate Cy42). 
Phylogenetic affinities of ArLV and relationships with RaLV. Phylogenetic analysis of the polyprotein amino acid sequences of representative viruses in the genera Brambyvirus, Bymovirus, Ipomovirus, Macluravirus, Potyvirus, Rymovirus, and Tritimovirus in the family Potyviridae clearly placed ArLV near to CYNMV, in the genus Macluravirus (Fig. 2; Table 1). Similarly close relationships were detected by Blast analysis with other macluraviruses, including Maclura mosaic virus (type member of the genus), Cardamom mosaic virus, and Narcissus latent virus (not shown). As observed already for CYNMV, the present analysis also confirmed that macluraviruses have their closest relationship in the family Potyviridae with the bymoviruses (Fig. 2).

However, the virus showing the highest homology in Blast analyses with the ArLV sequences was the Cy42 isolate of RaLV, for which a long sequence fragment $(5.8 \mathrm{~kb})$ is available (Ciuffo et al. 2011) (HQ449546). The RaLV-Cy42 sequence showed an overall 80.5\% nucleotide identity level with the ITBr05 isolate, the corresponding values with the FR37 and FR50 isolates being 81.9\% (Cy42-FR37) and 80.6\% (Cy42-FR50), respectively (Table 2). As indicated earlier, the FR50 isolate was closely related to ITBr05 (97.3\% identity for the almost complete genome sequence), while the FR37 was almost as divergent as the Cy42 isolate, with a whole genome identity with ITBR05 of $82.3 \%$. The corresponding values for the encoded proteins when comparing with the partial Cy42 RaLV sequence were $92.7 \%$ (ITBr05), 95.3\% (FR37), and 93.9\% (FR50). The FR50 polyprotein shows an overall $97 \%$ identity with that of ITBr05 (not shown) and the corresponding value for the FR37 polyprotein is $92.2 \%$ (Table 2). Individual comparisons for each encoded protein revealed high levels

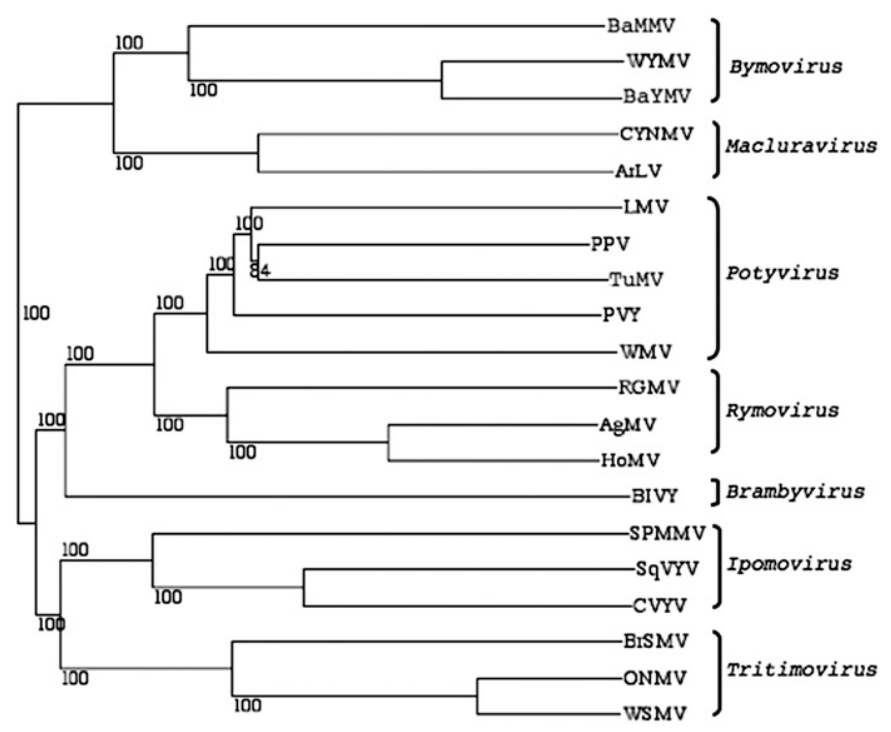

$\stackrel{0.05}{\longmapsto}$

Fig. 2. Unrooted phylogenetic tree reconstructed using the polyprotein of ArLV ITBR05 and of representatives of each genera in the family Potyviridae. The tree was obtained by the neighbor-joining method (Saitou and Nei 1987) run on the NJplot software, using a strict amino acid identity distance. Numbers at each node represent the percent bootstrap support (1,000 replicates). Branch lengths are proportional to genetic distances with the scale bar corresponding to 0.05 substitutions per amino acid site. The following viruses were used: Potato virus $Y$ (PVY, EU482153), Turnip mosaic virus (TuMV, AB440239), Plum pox virus (PPV KC347608), Watermelon mosaic virus (WMV, FJ823122), Lettuce mosaic virus (LMV, NC003605), Chinese yam necrotic mosaic virus (CYNMV, NC018455), Barley yellow mosaic virus (BaYMV, D01091), Wheat yellow mosaic virus (WYMV, D86634), Barley mild mosaic virus BaMMV, D83408), Wheat streak mosaic virus (WSMV AF057533), Oat necrotic mottle virus (ONMV, AY377938), Ryegrass mosaic virus (RGMV, Y09854), Hordeum mosaic virus (HoMV, AY623627), Cucumber vein yellowing virus (CVYV, AY578085), Squash vein yellowing virus (SqVYV, EU259611), Sweet potato mild mottle virus (SPMMV, Z73124), Brome streak mosaic virus (BrSMV, Z48506), Agropyron mosaic virus (AgMV, AY623626), and Blackberry virus Y (BlVY AY994084). of identity (91.7 to $98.7 \%$ ) (Table 1), with the exception of the CP, which was more divergent and showed only $81.4 \%$ identity with that of ITBR05 (see below). A phylogenetic analysis (Fig. 3) performed on a small, 939-nt sample overlapping the NIa and NIb genes for which sequence data were available for several other RaLV artichoke or Ranunculus isolates (Ciuffo et al. 2011) revealed the existence of three clusters of isolates, one composed of the five RaLV isolates sequenced by Ciuffo et al. (2011), four of them coming from globe artichoke (Cy42, Cy46, Cy49, and IFA235) and the other from Ranunculus (RN128). Another cluster was composed of the ITBr05 and FR50 artichoke isolates and the third of the FR37 artichoke isolate alone. In this short region, the average nucleotide divergence between these groups was 17.6 to $18.9 \%$, whereas the intragroup divergence was only of 2.6 to $2.9 \%$. A phylogenetic analysis based on a $2.1-\mathrm{kb}$ sequence fragment spanning the end of the NIb gene and extending to the end of the genome provided evidence for a similar grouping of isolates in three clusters (FR37, FR50 + ITBr05, and RaLV Cy42) grouping with the RaLV RN128 isolate from Ranunculus (result not shown).

Sequence comparisons between these five isolates using the $\mathrm{CP}$ gene alone showed nucleotide divergence levels of 24 to $24.1 \%$ between FR37 and FR50 and ITBr05, 19.3 to $19.7 \%$ between FR37 and $\mathrm{Cy} 42$ and the other RaLV isolate, and 25.2 to $26.6 \%$ between FR50 and ITBr05 and Cy42 and the other RaLV isolate (Table 2).

\section{DISCUSSION}

The results reported here represent the first complete genomic sequences for ArLV isolates, making ArLV the second macluravirus after CYNMV (Kondo and Fujita 2012) for which the full genome has become available. The ArLV identity of the ITBr05 isolate is based on several converging elements, including its detection by molecular hybridization with an ArLV-specific probe (Minutillo et al. 2012) and its reaction in ISEM with an ArLV-specific antiserum prepared more

TABLE 2. Whole-genome and coat protein (CP) nucleotide (nt) and encoded amino acid (aa) sequence identity levels between the different clusters of Artichoke latent virus (ArLV) and Ranunculus latent virus (RaLV) isolates

\begin{tabular}{cccc}
\hline & \multicolumn{3}{c}{ Sequence identity (\%) } \\
\cline { 2 - 4 } & $\begin{array}{c}\text { ArLV-FR37 vs. } \\
\text { ArLV-ITBr05 } \\
\text { and ArLV-FR50 }\end{array}$ & $\begin{array}{c}\text { ArLV-FR37 vs. } \\
\text { RaLV-Cy42 } \\
\text { and RaLV-RN128 }\end{array}$ & $\begin{array}{c}\text { ArLV-ITBr05 and } \\
\text { ArLV-FR50 vs. } \\
\text { RaLV-Cy42 and } \\
\text { RaLV-RN128 }\end{array}$ \\
\hline $\begin{array}{c}\text { Whole genome } \\
\text { nt }\end{array}$ & $82.1-82.3$ & $81.9^{\mathrm{a}}$ & $80.5-80.6^{\mathrm{a}}$ \\
aa & $92.2-94.5$ & $95.3^{\mathrm{a}}$ & $92.7-93.9^{\mathrm{a}}$ \\
CP & & & \\
nt & $75.9-76$ & $80.3-80.7$ & $73.4-74.8$ \\
aa & $80.2-80.8$ & $87.9-88.6$ & $79.7-82.1$ \\
\hline
\end{tabular}

a Only the partial 5.8-kb sequence of Cy42 was used for comparisons.

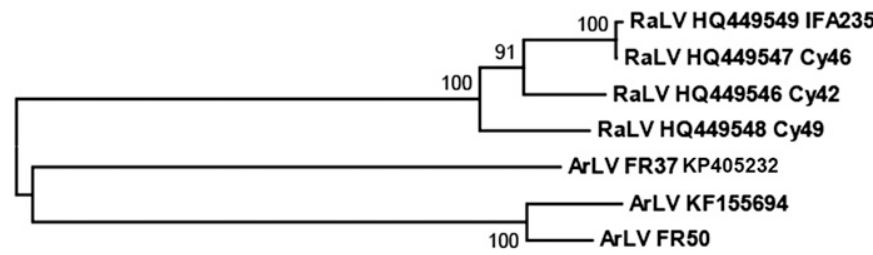

\subsection{2}

Fig. 3. Unrooted phylogenetic tree reconstructed using a partial approximately 939-nucleotide sequence overlapping the NIa and NIb genes of ArLV (this work) and RaLV (GenBank) isolates. The tree was obtained by the neighborjoining method using Mega 5.2 and using a strict amino acid identity distance. Bootstrap support (above 70\%, 1,000 replicates) is indicated. Branch lengths are proportional to genetic distances, with the scale bar corresponding to 0.02 substitutions per amino acid site. 
than 30 years ago. Although fewer elements are available, the identity of the FR50 isolate as an ArLV isolate is established by its reaction with the ArLV probe and by its high level of identity with the ITBr05 isolate. The situation of the FR37 isolate which, similarly to the RaLV isolates analyzed by Ciuffo et al. (2011), did not react in hybridization assays with the ArLV probe, is more complex and will be discussed below.

ArLV was proposed originally as a member of the genus Potyvirus on the basis of biological and physiochemical characteristics determined by technologies available at that time (Rana et al. 1982). The sequence data and overall genome organization determined in the present study for the ITBr05 and FR50 isolates show clearly that ArLV is a member of the genus Macluravirus, in agreement with the hypothesis of Ciuffo et al. (2011). Therefore, we propose to change the taxonomic position of ArLV and to move it among the macluraviruses.

The analysis of the ArLV genome sequence confirms many of the peculiarities previously identified for CYNMV (Kondo and Fujita 2012), including (i) a shorter genome, corresponding to the absence of a P1 protein-coding region and a divergent, shorter HC-Pro than in potyviruses; (ii) a short P3N-PIPO of only 56 aa; (iii) polyprotein cleavage sites with no clear consensus for positions -6 to -3 but a conserved $\mathrm{L}$ at position -2 and a $\mathrm{Q}(\mathrm{E}) / \mathrm{M}(\mathrm{A}, \mathrm{I})$ cleavage site consensus; and (iv) the absence of a DAG aphid-transmission triplet (López-Moya et al. 1999) in the N-terminal region of the CP (see below).

The findings of Ciuffo et al. (2011), which suggested frequent RaLV infection in artichoke, prompted speculations about a possible synonymy with ArLV. The currently accepted sequence-based species demarcation criteria for the family Potyviridae are nucleotide divergence of over $24 \%$ or amino acid divergence of over $20 \%$ for either the full genome or the CP gene (Adams et al. 2012). Although it lacks the $5^{\prime}$ genome part, comparison of the sequence of the Cy42 RaLV sequence with the ArLV sequences determined here provides some information about the relationships between ArLV and RaLV because the values obtained are clearly within the level of variability accepted for a species (Table 2). Completing the Cy42 sequence is unlikely to reverse this trend because the CI, 7K, and HC-Pro genes appear to be well-conserved genes when comparing ArLV isolates or when comparing ArLV with CYNMV (Table 1). The analysis of the CP gene, for which complete sequences are available for several isolates of ArLV and RaLV, yields similar results: in the majority of comparisons, divergence values within the species boundaries are observed, and those values above the cut-off are always close to it. Concerning isolate FR37, it is clearly divergent but, for all sequence-based criteria, it falls within the species boundaries when compared with ITBr05 or FR50 (Table 2). Therefore, it clearly belongs to the ArLV species. In addition, its polyprotein and its CP show identity levels when compared with the Cy42 and RN128 RaLV isolates that are also well within the $20 \%$ amino acid identity cut-off delimiting species. The results reported here also show that, when assayed in ISEM, ArLV (ITBr05) and RaLV showed strong serological cross-reactions (Fig. 1), another criterion of taxonomic significance. Fitting together these observations, the best conclusion is that RaLV should be considered as a strain (suggested name: Ranunculus strain) of ArLV and not as a distinct species. Thus far, all analyzed RaLV isolates appear to cluster together (Fig. 3) regardless of whether they were isolated from artichoke or from Ranunculus spp. Whether ArLV isolates such as ITBr05, FR50, or FR37 are able to infect Ranunculus spp. remains to be investigated; however, the fact that FR37 systemically infects $N$. clevelandii, a host previously reported to be differential between ArLV and RaLV (Ciuffo et al. 2011), suggests that the differences between the various strains of ArLV may not be as strong as previously thought. Given its significant divergence level, isolate FR37 is likely to represent another distinct strain within the ArLV species (Fig. 3).

An important practical point concerns the needed evolution of the diagnostic techniques in light of the present taxonomic clarification. In the experiments reported here, isolate FR37 could not be detected using the ArLV-specific molecular detection assay of
Minutillo et al. (2012), an observation that parallels the results of Ciuffo et al. (2011) on RaLV isolates (now Ranunculus strain of ArLV). This result is probably due to the high divergence of the various ArLV strains in the corresponding region. Therefore, this probe should be regarded as ArLV specific but also as unsuitable for the detection of all isolates and strains of ArLV. The same reasoning applies to the ArLV30F and ArLV500R primer pair (Ciuffo et al. 2011), suggesting a need for the development of novel ArLV-specific assays of broader polyvalence for use in artichoke certification programs.

The shorter HC-Pro, with its truncated N-terminal region, lacks, as for CYNMV (Kondo and Fujita 2012), all the motives known to be involved in systemic movement, silencing suppression, and aphid transmission. It is known that the approximately $100 \mathrm{~N}$-terminal amino acids of the HC-Pro are dispensable for systemic movement in some potyviruses (German-Retana et al. 2000) but such deletions clearly affect aphid transmissibility. ArLV is transmitted efficiently by aphids (Rana et al. 1982) and, although no information is available on its ability to interfere with RNA silencing, it readily systemically infects its artichoke or herbaceous experimental hosts such as $N$. benthamiana. Further analysis of the ArLV HC-Pro is needed to better understand the functions of macluraviruses, and their interactions with their hosts and vectors. The ability to suppress gene silencing proved to be a fundamental parameter in mixed infections between potyviruses and viruses belonging to other genera, possibly explaining synergistic effects. Although generally symptomless, ArLV has been reported to sometimes cause symptoms (Gallitelli et al. 2012), possibly as a consequence pf synergistic interactions with other viral agents. The discovery of a novel Comoviridae sp. and a novel Alphaflexiviridae sp. in the French symptomatic artichoke samples from which the FR37 and FR50 isolates were obtained supports such a scenario. AILV is a nepovirus that probably lacks an efficient suppressor of gene silencing (Santovito et al. 2014) but benefits from the HC-Pro of Potato virus $Y$ to establish a stable systemic infection in tobacco plants, which otherwise undergo recovery from viral symptoms. Due to the frequent occurrence of mixed infections between ArLV and AILV and to the symptoms observed in such plants, it would be interesting to study in the future the mechanisms of their interaction and, more broadly, if and how ArLV may interfere with its hosts' silencing machinery.

\section{ACKNOWLEDGMENTS}

We thank M. Turina, Istituto per la Protezione sostenibile delle piante, CNR, Turin, Italy for supplying RaLV isolate and antiserum and A. De Stradis, Istituto per la Protezione sostenibile delle piante, CNR, Bari, Italy for observations with the electron microscope. This work was supported by a grant of University of Bari (http://www.uniba.it), Italy in the framework of Research funds 2010 "Study of plant-pathogen interactions in Artichoke" grant ORBA10Q104.

\section{LITERATURE CITED}

Acquadro, A., Papanice, M., Lanteri, S., Bottalico, G., Portis, E., Campanale, A., Finetti-Sialer, M. M., Mascia, T., Sumerano, P., and Gallitelli, D. 2010. Production and genotyping of virus-free plants in a reflowering globe artichoke varietal type. Plant Cell Tiss. Org. Cult. 100:329-337.

Adams, M. J., Zerbini, F. M., French, R., Rabenstein, F., Stenger, D. C., and Valkonen, J. P. T. 2012. Family Potyviridae. Pages 1069-1089 in: Virus Taxonomy: Classification and Nomenclature of Viruses. A. M. Q. King, M. J. Adams, B. Eric, E. B. Carstens, and E. J. Lefkowitz, eds. Elsevier Academic Press, San Diego, CA.

Candresse, T., Marais, A., Faure, C., and Gentit, P. 2013. Association of Little cherry virus 1 with the Shirofugen stunt disease and characterization of the genome of a divergent LChV1 isolate. Phytopathology 103:293-298.

Chung, B. Y., Miller, W. A., Atkins, J. F., and Firth, A. E. 2008. An overlapping essential gene in the Potyviridae. Proc. Natl. Acad. Sci. USA 105: 5897-5902.

Ciuffo, M., Testa, M., Lenzi, R., and Turina, M. 2011. Ranunculus latent virus: A strain of Artichoke latent virus or a new macluravirus infecting artichoke? Arch. Virol. 156:1053-1057.

Gallitelli, D., Mascia, T., and Martelli, G. P. 2012. Viruses in artichoke. Adv. Virus Res. 84:289-324. 
German-Retana, S., Candresse, T., Alias, E., Delbos, R. P., and Le Gall, O. 2000. Effects of green fluorescent protein or b-glucuronidase tagging on the accumulation and pathogenicity of a resistance-breaking Lettuce mosaic virus isolate in susceptible and resistant lettuce cultivars. Mol. Plant-Microbe Interact. 13:316-324.

Grieco, F., and Gallitelli, D. 1999. Multiplex reverse-transcription polymerase chain reaction applied to virus detection in globe artichoke. J. Phytopathol. 147:183-185.

Hull, R. 2002. Matthews' Plant Virology. Academic Press, San Diego, CA.

Kondo, T., and Fujita, T. 2012. Complete nucleotide sequence and construction of an infectious clone of Chinese yam necrotic mosaic virus suggest that macluraviruses have the smallest genome among members of the family Potyviridae. Arch. Virol. 157:2299-2307.

López-Moya, J. J., Wang, R. Y., and Pirone, T. P. 1999. Context of the coat protein DAG motif affects potyvirus transmissibility by aphids. J. Gen. Virol. 80:3281-3288.

Lütcke, H. A., Chow, K. C., Mickel, F. S., Moss, K. A., Kern, H. F., and Scheele, G. A. 1987. Selection of AUG initiation codons differs in plants and animals. EMBO J. 6:43-48.

Marck, C. 1988. DNA Strider: A "C" programme for the fast analysis of DNA and protein sequences on the Apple Macintosh family computers. Nucleic Acids Res. 16:1829-1836.

Minutillo, S., Mascia, T., and Gallitelli, D. 2012. Development of a commercial diagnostic kit for the certification of the phytosanitary status in nursery production of globe artichoke. Eur. J. Plant Pathol. 134:459-465.

Rana, G. L., Russo, M., Gallitelli, D., and Martelli, G. P. 1982. Artichoke latent virus: Characterisation, ultrastructure and geographical distribution. Ann. Appl. Biol. 101:279-289.
Roberts, I. M., and Harrison, B. D. 1979. Detection of potato leafroll and potato mop-top by immunosorbent electron microscopy. Ann. Appl. Biol. 93:289-297.

Saitou, N., and Nei, M. 1987. The neighbor-joining method-A new method for reconstructing phylogenetic trees. Mol. Biol. Evol. 4:406-425.

Santovito, E., Mascia, T., Siddiqui, S. A., Minutillo, S. A., Valkonen, J. P. T., and Gallitelli, D. 2014. Infection cycle of Artichoke Italian latent virus in tobacco plants: meristem invasion and recovery from disease symptoms. PLoS One 9:e99446.

Savino, V., Gallitelli, D., Jankulova, M., and Rana, G. 1977. A comparison of four isolates of Artichoke Italian latent virus (AILV). Phytopathol. Mediterr. 16:41-50.

Tamura, K., Peterson, D., Peterson, N., Stecher, G., Nei, M., and Kumar, S. 2011. MEGA5: molecular evolutionary genetics analysis using maximum likelihood, evolutionary distance, and maximum parsimony methods. Mol. Biol. Evol. 28:2731-2739.

Thompson, J. D., Higgins, D. G., and Gibson, T. J. 1994. CLUSTALW: improving the sensitivity of progressive multiple sequence alignment through sequence weighting, position-specific gap, penalties and weight matrix choice. Nucleic Acids Res. 22:4673-4680.

Thompson, S., Fraser, R. S. S., and Barden, K. L. 1988. A beneficial effect of trypsin on the purification of Turnip mosaic virus (TuMV) and other potyviruses. J. Virol. Methods 20:57-64.

Turina, M., Ciuffo, M., Lenzi, R., Rostagno, L., Mela, L., Derin, E., and Palmano, S. 2006. Characterization of four viral species belonging to the family Potyviridae isolated from Ranunculus asiaticus. Phytopathology 96: $560-566$ 